\title{
Necesidades de las personas adultas mayores que hacen uso de los servicios del sector hotelero de 4 y 5 estrellas en la Ciudad de Puebla
}

\author{
Needs of older adults who make use of the services of 4 and 5 star hotel sector in the City of \\ Puebla
}

\author{
Roberto C. Hernández-Reyes ${ }^{a}$, Aline Rivas-Martínez ${ }^{b}$, Myriam Coronado-Menese ${ }^{c}$, Maribel \\ González-Cadena ${ }^{d}$
}

\begin{abstract}
:
The present work, which identifies the needs of older adults who make use of the services of the 4- and 5-star hotel sector in the city of Puebla, has a non-experimental transverse design type with descriptive scope.

According to research, Gerontological tourism is considered an emerging segment due to the increase in its population and changes in their needs and this segment is part of the objectives of the National and Sectoral Development Plan manifesting achieve an accessible and inclusive Mexico to allow an active aging.

The intercensal survey conducted by the INEGI indicates that the places with higher rate of older adults in the country are Iztapalapa, Guadalajara, Gustavo A. Madero Delegation and Puebla. In this context, in the first one, there is a lack of tourism by its insecurity index (Vega, 2015); the State of Jalisco has already taken actions to be an inclusive and accessible State, the Gustavo A. Madero Delegation serving older adults providing them a card to visit tourist destinations.

This work is carried out in the city of Puebla since it is considered a Historical Heritage of Humanity City with a vast range of tourist where in addition to having a circuit that people with disabilities can visit it. In the documentary investigation, there is not programs, so much in the private and public sector, which attend to the tourist needs of the segment studied.
\end{abstract}

Because of the research, there are infrastructure in the 4- and 5-star hotels that must be attended to offer a better service to the older adult, beside training service who work in them, mainly from treatment a physical and cultural activities.

\section{Keywords:}

Gerontology, gerontology tourism, distinctive G, Hotel infrastructure, elderly tourists

\section{Resumen:}

El presente trabajo, que identifica las necesidades de las personas adultas mayores que hacen uso de los servicios del sector hotelero de 4 y 5 estrellas en la ciudad de Puebla, posee un diseño no experimental de tipo transversal con alcance descriptivo.

De acuerdo a investigación, el turismo gerontológico se considera un segmento emergente debido al incremento de su población y a los cambios en sus necesidades y, este segmento, forma parte de los objetivos del Plan Nacional y Sectorial de Desarrollo que manifiestan lograr un México accesible e incluyente para permitir un envejecimiento activo.

La encuesta intercensal realizada por el INEGI indica que los lugares con superior índice de personas adultas mayores en el país son Iztapalapa, Guadalajara, delegación Gustavo A Madero y Puebla; en este contexto, en el primero existe falta de turismo por su índice

\footnotetext{
a Carlos R. Hernández Reyes, Universidad Autónoma del Estado de Hidalgo, Escuela Superior de Tizayuca, Email: alumnoroberto@outlook.es

b Aline Rivas Martínez, Universidad Autónoma del Estado de Hidalgo, Escuela Superior de Tizayuca, Email: alinervs@outlook.es

c Myriam Coronado Meneses, Universidad Autónoma del Estado de Hidalgo, Escuela Superior de Tizayuca, Email: myriam_coronado3187@uaeh.edu.mx

Maribel Cadena González, Universidad Autónoma del Estado de Hidalgo, Escuela Superior de Tizayuca, Email: maribel_gonzalez4257@uaeh.edu.mx
} 
de inseguridad (Vega, 2015), el estado de Jalisco ya ha tomado acciones para ser una entidad federativa incluyente y accesible, en la delegación Gustavo A Madero se atiende a las personas adultas mayores proporcionándole una tarjeta para visitar destinos turísticos.

Este trabajo se realiza en la Ciudad de Puebla ya que, aunado a lo descrito, se considera una ciudad Patrimonio Histórico de la Humanidad con una vasta oferta turística donde, además de tener un circuito que pueden visitar las personas con discapacidad, en la investigación documental no se encuentran programas, tanto las instituciones del sector público como del privado, que atiendan las necesidades turísticas del segmento en estudio.

Como resultado de la investigación, existen factores de infraestructura en los hoteles de 4 y 5 estrellas que deben atenderse para brindar un mejor servicio a los adultos mayores así de capacitación a los prestadores de servicio que laboran en ellos, principalmente de trato y de actividades físicas y culturales.

\section{Palabras Clave:}

Gerontología, Turismo Gerontológico, Distintivo G, Infraestructura Hotelera, Turista de la tercera edad

\section{Introducción}

Este apartado brinda el contexto de la investigación en el planteamiento del problema, la justificación y el objetivo que línea este trabajo.

\subsection{Planteamiento del problema}

El Instituto Nacional de Estadística, Geografía e Informática (INEGI, 2016) indica a la ciudad de Puebla como el cuarto lugar con un índice de 115,096 personas adultas mayores.

En este contexto, de acuerdo con la Procuraduría General de Justicia del Distrito Federal (PGJDF), la delegación de Iztapalapa, que ocupa el primer lugar, tiene el mayor índice de inseguridad lo que es un factor que determina la falta de turismo en la delegación (Vega, 2015); por su parte, el estado de Jalisco, con el segundo lugar, ha tomado acciones para ser accesible con la primera playa y pueblo incluyente en México (Rodríguez, 2015); de igual forma, el gobierno de la delegación Gustavo A Madero proporciona a las personas adultas mayores una tarjeta para visitar destinos turísticos entre los que se encuentran las Trajineras de Xochimilco, La villa, el Centro Histórico de Coyoacán, Guerrero, Puebla y el Estado de México (Flores, 2014).

Esta investigación se realiza en la Ciudad de Puebla ya que, además de poseer uno de los primeros lugares con alto índice de personas adultas mayores, es considerada como una ciudad Patrimonio Histórico de la Humanidad al contar con una vasta oferta turística y que, aunado a ello, la investigación documental realizada indica que las instituciones, tanto del sector público como del privado, no cuentan con programas que atiendan las necesidades turísticas de este segmento (Diario, Intolerancia, 2015).

\subsection{Justificación}

Tanto en los objetivos nacionales como los sectoriales en materia turística, señalan reconocer y atender las necesidades de las personas adultas mayores a fin de lograr un México accesible e incluyente.

La investigación documental exhibe la falta de accesibilidad para personas adultas mayores en varios lugares del país.

En este contexto, la Secretaria de Turismo junto con la Asociación de Hoteles y Moteles y el Instituto Nacional de Atención a las Personas Adultas Mayores, han otorgado el distintivo $G$ a empresas turísticas en la ciudad de Villahermosa en el estado de Tabasco por cumplir las características necesarias en el sector turístico, además de ofrecerles capacitación a los prestadores de servicios para poder atender las necesidades de las personas adultas mayores y a la accesibilidad de los servicios turísticos.

No obstante, la ciudad de Puebla cuenta con una extensa oferta turística y, de acuerdo con el INEGI, ocupa el cuarto lugar con población de adultos mayores, para sus instituciones del sector público y privado, las necesidades en cuanto a accesibilidad e inclusión del Turismo Gerontológico no tienen mayor relevancia ya que carecen de programas o propuestas suficientes que las atiendan. Expuesto lo anterior, se considera relevante realizar una investigación documental que determine las necesidades de las personas adultas mayores en el sector hotelero con clasificación 4 y 5 estrellas la Ciudad de Puebla

\subsection{Objetivo General}

Identificar las necesidades de las personas adultas mayores que hacen uso de los servicios del sector hotelero de 4 y 5 estrellas en la ciudad de Puebla con la realización de investigación documental y de campo que permita reconocer y realizar propuesta para atender necesidades.

1.4. Materiales y Métodos

Este trabajo posee un diseño no experimental de tipo transversal con alcance descriptivo para cumplir con el objetivo de investigación. 
En la primera etapa se realizó investigación documental con fuentes de información y páginas de internet de organizaciones oficiales.

En la segunda etapa se diseñó un instrumento de investigación y se presenta información primaria con base al análisis de resultados de su aplicación, en el mes de abril de 2018, a personas adultas mayores para conocer sus necesidades al hacer uso de servicios de hotelería en la Ciudad de Puebla.

\section{Marco conceptual}

\subsection{Turismo}

Hunziquer y Krapf (citados por Guerrero y Ramos, 2011) definen al Turismo como "la totalidad de las relaciones y fenómenos generados por el viaje y la estancia de forasteros, siempre y cuando la estancia no implique el establecimiento de una residencia permanente".

\subsection{Gerontología}

La gerontología se define como la ciencia que estudia el envejecimiento en todos sus aspectos e incluye las ciencias biológicas y médicas, psicológicas y sociológicas; además, de la aplicación del conocimiento científico en beneficio del envejecimiento y de los adultos mayores (Psziemiarower \& Psziemiarower, 1992).

\subsection{Turista de la Tercera edad}

Según la Organización Mundial de la Salud (OMS) las personas de 60 a 74 años son considerados de edad avanzada, de 75 a 90 años viejas o ancianas y los que pasan los 90 años se les denomina grandes, viejos o longevos. A todo individuo mayor de 60 años se le llamará de forma indistinta persona de la tercera edad (Guzmán, 2010).

\subsection{Distintivo G}

Es un Sistema de Gestión que profesionaliza a aquellas empresas cuyo segmento de clientes sea la población Adulta Mayor y es avalado por el Instituto Nacional de Atención a las Personas Adultas Mayores (INAPAM). El distintivo $\mathrm{G}$, en Turismo Gerontológico, se crea en el año 2009 como un Sistema de Gestión para la Profesionalización y Estandarización en empresas del ramo turístico para la atención de las personas adultas mayores a través del esparcimiento y la diversión.

Actualmente el Distintivo G está enfocado en crear rutas de accesibilidad tanto para las personas adultas mayores como para personas con capacidades diferentes.

\section{Marco referencial}

3.1 Turismo gerontológico en la Ciudad de Puebla

En la ciudad de Puebla se encuentran ubicados dos centros de Gerontología dependientes del Desarrollo
Integral de la Familia (DIF) que tienen como objetivo promover la integración social de los adultos mayores de 60 años.

Uno de los principales servicios que ofrecen son actividades Integrales como actividades físicas, recreativas y culturales con el fin de mejorar la calidad de vida de las personas de la tercera edad, para lograr una mejor convivencia en su entorno social y familiar.

Entre los eventos que se han organizado en Puebla a favor de los adultos mayores se encuentran los Juegos Estatales Deportivos y Culturales de la Tercera Edad, que se celebran en los primeros días de mayo, además de otras actividades distintas como natación, atletismo, baile de salón y otros eventos como torneos de ajedrez y canto. Además de estas actividades que los mantendrán activos, también se encuentran inscritos al INAPAM, lo cual les da derecho a descuentos en salud, transporte, cultura y recreación." (Adultos Mayores, 2016).

\subsection{Sector hotelero en la Ciudad de Puebla}

Como lo muestra la Tabla 1 la oferta hotelera en el estado de Puebla está compuesta por 745 hoteles con 20,517 habitaciones de los cuales se destaca que el $30 \%$ del total de los hoteles se encuentra en la capital del estado aglutinando la Ciudad de Puebla el $51 \%$ de la oferta en cuartos (Sistema De Información Turística Estatal, 2015).

Tabla 1: Oferta Hotelera en el estado de Puebla

\begin{tabular}{lrcc}
\hline \multicolumn{2}{c}{ Categoría } & Hoteles & Habitaciones \\
\hline Estrellas & 4 & 37 & 3,105 \\
Estrellas & 5 & 31 & 4,959 \\
\hline
\end{tabular}

Fuente: Puebla, 2015.

4. Lineamientos nacionales y estatales en el ámbito turístico

4.1 Lineamientos nacionales

El Plan Nacional de Desarrollo 2013-2018 reconoce que el turismo representa una alternativa real para la generación de empleos, incrementar los mercados donde operan las pequeñas y medianas empresas, así como la posibilidad de preservar la riqueza natural y cultural del país. En este sentido, las políticas de desarrollo del sector deben considerar criterios enfocados a incrementar la contribución del turismo a la reducción de la pobreza y la inclusión social (Plan Nacional de Desarrollo, 2013).

En el Programa Sectorial de turismo 2013-2018 se reitera el compromiso del Gobierno Federal para crear instrumentos que hagan del Sector una industria incluyente, que sirva como una herramienta para la democratización de la productividad, consolidando el modelo turístico basado en criterios de sustentabilidad social, económica y ambiental, velando siempre por el cuidado y preservación del patrimonio cultural, histórico y 
natural del país, convirtiendo al turismo en fuente de bienestar social (Programa Sectorial de Turismo, 2013)

\subsection{Lineamientos del estado de Puebla}

De acuerdo a la Ley de Turismo del Estado de Puebla los integrantes de la Quincuagésima Séptima Legislatura Local suscribieron la agenda legislativa 2008-2011. Dicho marco jurídico establece las bases para la planeación, programación y evaluación de las políticas públicas en materia turística en el Estado y sus Municipios que busca el beneficio social.

Dentro del Programa Estatal de Turismo se incorpora al Turismo Accesible y de Adultos Mayores con la intención de que la Secretaría, con el apoyo y en coordinación de las dependencias y entidades competentes, promueva la prestación de los servicios turísticos con accesibilidad.

Los prestadores de servicios turísticos, organizaciones o asociaciones de los sectores sociales o privados, así como los Ayuntamientos, podrán solicitar a la Secretaría que un área, Municipio o región sea declarado Zona de Desarrollo Prioritario para que, en su caso, puedan recibir los apoyos que contemple las Secretaría dentro del Programa Estatal de Turismo $(\mathrm{H}$. Congreso del estado de Puebla, 2013).

\section{Investigación de campo}

\subsection{Población y muestra}

En atención al tema y al objetivo de investigación, la población se constituyó por personas adultas mayores pensionadas/jubiladas que puedan viajar.

De acuerdo al reporte del periódico el Financiero (2015) la población de adultos mayores es de 11,7 millones y el porcentaje de pensionadas/jubiladas que puede viajar es del 9,6\%, siendo $1^{\prime} 053,000$ de personas de los cuales, el $2.5 \%$ tiene como destino preferido viajar a Puebla siendo el equivalente de 26, 325 personas adultas mayores El cálculo del tamaño de la muestra en esta investigación se supedita a tres factores:

1. El porcentaje de confianza con el que se desea generalizar los datos en la población total.

2. El porcentaje de error que se está dispuesto a aceptar en la investigación.

3. El nivel de variabilidad que se calcula para la comprobación de la hipótesis.

El tipo de muestreo de esta investigación es probabilístico con población finita, la técnica de muestreo que se toma es aleatoria. La clase de nuestra investigación es de tipo probabilística, siendo conocido el número de nuestra población finita.
Para determinar el tamaño de la muestra en poblaciones finitas se adoptaron las recomendaciones Guzmán (2010) y se optó por tolerar un porcentaje de error del $5 \%$ con un nivel de confianza del 95\% para aplicar en la ecuación propuesta por el autor.

Al aplicar la ecuación se determinó el tamaño de la muestra como se aprecia en la Tabla 2

\section{Tabla 2: Muestra de unidades de análisis para aplicación del instrumento}

\begin{tabular}{cccc}
\hline Unidades de análisis & $\begin{array}{c}\text { Tamaño de } \\
\text { la muestra }\end{array}$ & $\begin{array}{c}\text { Porcentaje } \\
\text { válido de la } \\
\text { muestra }\end{array}$ \\
\hline 26,325 & $\begin{array}{c}\text { Adultos } \\
\text { mayores }\end{array}$ & 133 & $100 \%$ \\
\hline
\end{tabular}

Fuente: elaboración propia

El instrumento aplicado posee un intervalo de confianza del $95 \%$, y una consistencia interna de 0.90 en el instrumento de investigación con el coeficiente Alfa de Cronbach, lo que constató la fiabilidad del instrumento de investigación.

\subsection{Principales resultados}

En esta sección el procedimiento que se utiliza para construir el instrumento de investigación, el cual se supedita a los lineamientos para obtención del Distintivo $G$ de la infraestructura hotelera.

A continuación, se presentan, de manera descriptiva, los resultados del instrumento aplicado para reconocer las necesidades de las personas adultas mayores que hacen uso de los servicios del sector hotelero de 4 y 5 estrellas en la ciudad de Puebla.

Respecto a la ocupación del adulto mayor los resultados muestran que el $58 \%$ de los encuestados se encuentra pensionado seguido del $35 \%$ de adultos mayores que trabaja y el resto (7\%) no contestó.

Al preguntarles cuales eran los lugares de interés más importantes dentro de las opciones de la encuesta, se encontró que el $30 \%$ de los adultos mayores prefiere destinos de sol y playa y naturaleza, respectivamente; el $24 \%$ le interesa visitar ciudades culturales y al $16 \%$ le gusta visitar pueblos.

Al preguntarles si conocen el Distintivo G el $84 \%$ mostró grado de desconocimiento del tema mientras que el $14 \%$ comentó si conocer al respecto y el $2 \%$ no contestó.

Respecto a los lineamientos para el otorgamiento de Distintivo G, y al objetivo de esta investigación, las principales necesidades que los adultos mayores consideran debe poseer un hotel son, de mayor a menor porcentaje:

1. Pisos sin desniveles.

2. Baños acondicionados con pasamanos. 
3. Instalaciones para silla de rueda y bastón.

4. Áreas de esparcimiento para el adulto.

5. Actividades culturales y sociales.

6. Información de puntos de interés turísticos.

7. Servicio de reacondicionamiento físico.

Al preguntarles si se encontraban satisfechos con los servicios recibidos en el hotel donde se hospedaron, la mayoría dijo que si, aunque sugieren el mejoramiento de las instalaciones para poder sentirse libres y sin algún obstáculo que perjudique su integridad física, así como la mejora de capacitación correcta a los prestadores de servicios del sector hotelero para atender sus necesidades.

Por último, al preguntarles si el hotel donde se hospedan cumple con la infraestructura adecuada, en promedio el $70 \%$ contestó que no.

\section{Conclusiones}

Los resultados de esta investigación conducen a las siguientes conclusiones:

Con respecto a la ocupación, seis de cada diez adultos mayores se encuentran pensionados.

En atención al grado de conocimiento del Distintivo $\mathrm{G}$ en el sector hotelero, sólo uno de cada diez sabe al respecto. Una vez aplicado el instrumento de investigación, las principales necesidades de las personas adultas mayores que se hospedaron en hoteles de 4 y 5 estrellas de la Ciudad de Puebla son principalmente de infraestructura con la necesidad de pisos sin desniveles, con pasamanos e instalaciones para sillas de ruedas, bastones 0 andaderas en sus diferentes áreas así como de capacitación a los prestadores de servicio en el trato y actividades de reacondicionamiento físico y de la organización de culturales y sociales para el adulto mayor.

Derivado de la investigación documental y de campo, se recomienda a las autoridades de la Ciudad de Puebla atender lineamientos del Plan Nacional de Desarrollo, las políticas de desarrollo del sector y considerar criterios enfocados a incrementar la contribución del turismo a la inclusión social. Además de que, dentro de su Programa Estatal de Turismo, se determinó incorporar entre otros aspectos al Turismo Accesible y de Adultos Mayores con la intención de que la Secretaría, con el apoyo y en coordinación de las dependencias y entidades competentes, promueva la prestación de los servicios turísticos con accesibilidad.

\section{Agradecimientos}

Este trabajo ha sido realizado, en su mayoría, con la investigación de alumnos de la Licenciatura en Turismo durante su formación entre los semestres octavo a noveno.

\section{Referencias}

Adultos Mayores. (2016). Noticias adultas mayores. Recuperado el 2017, de http://www.adultos-mayores.net/en-puebla-se-llevan-a-caboacciones-para-los-adultos-mayores/

Diario, Intolerancia. (13 de 08 de 2015). Intolerancia Del político informado. Recuperado el 10 de 05 de 2017, de ciudad: http://intoleranciadiario.com/detalle_noticia/135793/ciudad/pueblamunicipio-pionero-en-fomentar-el-turismo-inclusivo-dinorah-lopezde-gali

Flores, A. C. (26 de 08 de 2014). La Jornada. Recuperado el 10 de 05 de 2017, de Capital: www.jornada.unam.mx/2014/08/26/capital/033n1cap

Gúzman, A. Q. (11 de 2010). UAEH. Recuperado el 23 de 05 de 2017, de https://www.uaeh.edu.mx/nuestro_alumnado/esc_sup/actopan/licenciat ura/Analisis\%20de\%20la\%20calidad\%20de\%20vida.pdf

Inapam. (2014). Instituto Nacional de las Personas adultas mayores. Recuperado el 2017, de www.inapam.gob.mx

Inegi. (2015). Encuesta Intercesal 2015. República Mexicana

Poder Ejecutivo Federal. (2013). Plan Nacional de Desarrollo 2013-2018. Recuperado de http://pnd.gob.mx/

Programa Sectorial de Turismo. (2013). Secretaria de Turismo. Recuperado el 2017

Psziemiarower, S., \& Psziemiarower, N. P. (1992). Asociacion gerontologica de Argentina. Buenos Aires.

Rodríguez, L. (13 de 02 de 2015). Secretaria de Infraestructura y Obra Pública. Recuperado el 08 de 05 de 2017, de Gobierno del Estado de Jalisco: siop.jalisco.gob.mx/prensa/noticia/580

Sistema De Información Turística Estatal. (2015). Gobierno de Puebla. Recuperado el 2017, de www.estadodepuebla.gob.mx

Vega, M. (2015). de Capital La Jornada. Recuperado el 08 de 05 de 2017 de: www.jornada.unam.mx/2015/12/31/capital/027ni1cap

\section{Apéndice A. Primer Apéndice}

\section{Cuestionario para las personas de la tercera edad}

El presente cuestionario se diseña para conocer el motivo que tienen las personas adultas mayores para hacer uso de los servicios de hospedaje, así como los intereses que tiene para viajar a la Ciudad de Puebla

La información que se obtenga de esta investigación es de carácter estrictamente confidencial y los datos resultantes se abordan con una óptica exclusivamente académica.

Instrucciones: Se le solicita proporcione usted los datos requeridos por este instrumento e indique, señale o describa la opción correspondiente a cada pregunta. 
1. Lugar de procedencia

2. Ocupación

2.1 Pensionado__ 2.2 Jubilado_ 2.3 Actualmente trabaja

3. Lugares de Interés

a) Ciudad

b) Sol y Playa

c) Naturaleza

d) Pueblos

4. ¿Realiza algún tipo de actividad recreativa o cultural? $4.1 \mathrm{Si}$ 4.2 No

5. ¿Pertenece algún club, organización o asociación?

$5.1 \mathrm{Si}$ $5.2 \mathrm{No}$

6. ¿Conoce acerca del distintivo $\mathrm{G}$ ?

a) $\mathrm{Si}$

b) $\mathrm{No}$

7. A continuación se enlistan las características del distintivo $G$ señale si el hotel donde se hospeda cuenta con alguna de ellas.

\begin{tabular}{|c|c|c|}
\hline & $\mathrm{Si}$ & No \\
\hline $\begin{array}{lcccc}\begin{array}{l}\text { Rampas en los accesos } \\
\text { establecimiento }\end{array} & & & \text { del } \\
\end{array}$ & & \\
\hline $\begin{array}{l}\text { Persona encargada de abrir y cerrar la } \\
\text { puerta }\end{array}$ & & \\
\hline Pesos sin desniveles & & \\
\hline $\begin{array}{l}\text { Baños acondicionados con pasamanos en } \\
\text { sus diferentes áreas }\end{array}$ & & \\
\hline $\begin{array}{l}\text { Baños con pisos antiderrapantes en el } \\
\text { área de regadera }\end{array}$ & & \\
\hline Iluminación en todas sus áreas & & \\
\hline $\begin{array}{l}\text { Instalaciones para sillas de ruedas, } \\
\text { bastones ó andaderas }\end{array}$ & & \\
\hline Señalizaciones luminiscentes visibles & & \\
\hline Pasamanos o rampas & & \\
\hline $\begin{array}{l}\text { Áreas de esparcimiento para el adulto } \\
\text { mayor }\end{array}$ & & \\
\hline $\begin{array}{l}\text { Actividades culturales y sociales para el } \\
\text { adulto mayor }\end{array}$ & & \\
\hline $\begin{array}{l}\text { Información que indique los puntos de } \\
\text { interés turísticos }\end{array}$ & & \\
\hline $\begin{array}{l}\text { Colaboradores que conocen el } \\
\text { reacondicionamiento físico que requiere } \\
\text { el adulto mayor }\end{array}$ & & \\
\hline
\end{tabular}

8. ¿El hotel en el que se hospeda cumple con la infraestructura adecuada para usted?

a) $\mathrm{Si}$

b) No

9. ¿Está satisfecho con el servicio durante su estancia en el hotel?
a) Muy satisfecho
b) Satisfecho
c) Poco satisfecho
d) Nada satisfecho

10. ¿Es importante para usted que los prestadores de servicios estén capacitados para atender a las personas adultas mayores?
a) Muy importante
b) Importante
c) Poco importante

¡Gracias por su atención! 